\title{
QUANTUM WEB OF GRAVITY
}

\author{
Eugene Machusky \\ Kyiv Polytechnic Institute, Ukraine \\ sivera@ukr.net
}

\begin{abstract}
The recently proposed and logically developed paradigm of deep genetical affinity of scientific terms "energy-entropy-information" harmonizes classical and quantum physics with general and special theories of relativity. For the first time in engineering practice, a complex vector-tensor-matrix description of gravitational, thermal and electromagnetic fields in ideal solid, liquid and gaseous media was performed analytically and without preliminary measurements. The basic set of fundamental quantum constants of the standard physical model has been computed with an extreme precision of $1 / 10^{\wedge} 64$, which is a natural limit arising due to elementary recursive arithmetic and elementary functional analysis in digital notation.
\end{abstract}

\section{KEYWORDS}

Quantum metrics; Quantum Information processing; Informational entropy

\section{INTRODUCTION}

The phenomenal mathematical efficiency of quantum field theories in modern high-speed information and communication systems has a simple explanation from the point of view of common sense and higher mathematical logic: both the quantum metric, and quantum physics, and quantum cosmology in their origin can be considered as universal self-information systems based on symbolic functional analysis and elementary recursive arithmetic of the relative parameters of the wave motion of matter in space and time.

Both classical and quantum physics arose as a result of numerous careful and long-term measurements, symbolic functional description and calculations of the observed trajectories of satellites, planets, stars, constellations, galaxies in cosmic space, as well as the observed parameters of the motion of atoms and subatomic particles in the inner space of electromagnetic accelerating systems with subsequent matching of heat transfer, charge transfer and mass transfer using energy diagrams developed for electrodynamics on the mathematical basis of classical thermodynamics.

Classical thermodynamics is based on two ideal wave functions of space and time - transcendental numbers "pi" and "e", but classical electrodynamics includes one more combined space-time number "c" a stroboscopic unit of rotational speed, which was obtained experimentally (read - approximately), but later voluntarily established by the scientific community as a universal metric unit of measurement 299792458 meters per second. In fact, such a combined unit - the ratio of metric units of space and time leads to a logical confusion of different and, therefore, fundamentally nonequivalent scientific categories "distance" and "duration", and this naturally and inevitably generates information uncertainty (entropy) between other parameters of motion - temperature (vibrational speed), progressive velocity (translational speed) and the corresponding energy, frequency, wavelength, momentum, acceleration, force, pressure.

Artificially established in the SI measurement system, the "exact" unit of speed with a decimal length of nine digits can be considered quite suitable for macroscale communications, but at the same time limits the mutual agreement of thermodynamics and electrodynamics to the nanoscale $10^{\wedge}(-9)$, the electronic level, which is six orders of magnitude higher than the level of nucleons $10^{\wedge}(-15)$. The second artificially set in $\mathrm{SI}$ "exact" metric unit of temperature $\mathrm{K}=2.7316$ naturally and inevitably limits the measurement accuracy at the level of $1 / 10^{\wedge} 5$. At the same time, the photon energy is determined experimentally with an 
accuracy of $10^{\wedge}(-34)$. In this situation, mathematical logic and the logic of common sense tell us that for mutual informational agreement of four different branches of physics (thermodynamics, electrodynamics, chromodynamics and gravidynamics it is necessary to establish the set of interconnected but different reference units of speed and temperature, but, as it turned out this is already a mathematical problem of minimizing the informational entropy of symbolic and numerical functional analysis.

Thus, the main logical error of modern metrology and related field theories is that fractional numbers are treated as mathematical constants. Elementary recursive arithmetic (sum and difference, product and ratio, exponentiation and root extraction) clearly shows that only integers, their sums and products always are true constants, since all their ratios and roots are in fact variables - these are infinite periodic (irrational), finite aperiodic (rational) and infinite aperiodic (transcendental) digital waves, which naturally and inevitably cause the manifestation of interference, superposition, dispersion, diffraction, refraction of the wave motion parameters - wavelength, temperature, frequency, amplitude, phase, polarization angle. But all this becomes clearly visible only at very small scales of spatial gradients and time intervals. Quite surprisingly but, as it turned out, only eight simple interrelated parametric equations containing as the variables only decimal length of the mantissa of transcendental numbers determine the quantum limits of informational entropy of continuous and discrete functional analysis and agree classical physics with special and general relativity [1]-[13].

\section{QUANTUM ARITHMETICAL KEYS TO METRICS MANIFOLD}

The two basic (polynomial $\mathrm{C}$ and exponential $\mathrm{X}$ ) quantum arithmetic keys to the topology and metrics of outer cosmic space and inner subatomic space are presented below by the recently derived parametric equation for the speed of light and the old and well-known equation for irradiation of absolute black body:

$$
\begin{aligned}
& C=\left(R / 10^{\wedge} 8+4^{*} \mathrm{pi}^{\star} \mathrm{C} / 10^{\wedge} 18\right)^{\wedge} 64^{*} 10^{\wedge} 7, \text { where } \mathrm{R}=\operatorname{Integer}\left\{10^{\wedge} 8^{*}\left(\mathrm{C} / 10^{\wedge} 7\right)^{\wedge}(1 / 64)\right\}=105456978, \\
& C=[299792457.86759133843368398914990500927337258665405914040533114633], \\
& \mathrm{L}^{*} \mathrm{~T}=\mathrm{h}^{\star} \mathrm{C} /\left(\mathrm{k}^{*} \mathrm{X}\right) \text { or } 1=\mathrm{h}^{\star} \mathrm{C} /\left(\mathrm{k}^{*} \mathrm{~L}^{*} \mathrm{~T}^{\star} \mathrm{X}\right)=\left(\mathrm{h}^{*} \mathrm{~F}\right) /\left(\mathrm{k}^{*} \mathrm{~T}\right) / \mathrm{X}, \text { where } \mathrm{F}=(\mathrm{C} / \mathrm{L}), \mathrm{X}=\operatorname{Root}\left\{\mathrm{X}^{*} \mathrm{e}^{\wedge} \mathrm{X} /\left(\mathrm{e}^{\wedge} \mathrm{X}-1\right)=5\right\}, \\
& X=[4.9651142317442762999999999999999999999999999999999999999999999999 \ldots],
\end{aligned}
$$

where $L$ - instant wavelength, $T$ - instant temperature, $h$ - instant unit of Planck, $C$ - instant unit of Maxwell, $\mathrm{k}$ - instant unit of Boltzmann, $\mathrm{F}$ - instant frequency. Accurately calculated value of $\mathrm{C}$ is rational number - a finite aperiodic decimal fraction, and accurately calculated value of $X$ is transfinite number an infinite aperiodic decimal fraction. As it turned out, this information is sufficient to determine analytically parameters of matter motion at subatomic scales. The presented manifold of interconnected matrices clearly demonstrates the geometric and arithmetic origin of fundamental quantum units from the Gaussian argument of the information entropy of the normal distribution Sqrt $\left(2^{*} \mathrm{pi}^{*} \mathrm{e}\right)$ :

Field of radius-vectors $\left.[R]=1+2 / 100^{*}\left(e+[A]^{*}\left(1+\operatorname{Sqrt}\left(2^{*} \mathrm{pi}^{*} \mathrm{e}\right) / 10\right)\right)\right)-$ radial matrix of Dirac.

Field of radial shift $\left.[\mathrm{A}]=\left(100^{*}([\mathrm{R}]-1) / 2-\mathrm{e}\right) /\left(1+\mathrm{Sqrt}\left(2^{*} \mathrm{pi}{ }^{*} \mathrm{e}\right) / 10\right)\right)-$ eccentricity matrix of Sommerfeld.

Field of perimeters $[P]=2^{*} \mathrm{PI}^{*}[\mathrm{R}]-$ informational entropy matrix of Planck-Heisenberg-Dirac.

Field of density of perimeters $[G]=[P]^{*}(1+[A])$ - gravitational matrix of Newton.

Field of amplitudes $[\mathrm{V}]=[\mathrm{R}]^{\wedge} 64^{*} 10^{\wedge} 7-$ translational velocity matrix of Maxwell.

Field of phases $[K B]=\operatorname{Cos}(12-[A] / 10)-\operatorname{Sin}(12-[A] / 10)-$ polarization angle matrix of Boltzmann .

Field of entropy $[N A]=100^{*}\left[\right.$ Sqrt $\left.\left(8^{*} \mathrm{pi}^{\star} \mathrm{e} /\left(8^{*} \mathrm{pi}^{*} \mathrm{e}+137^{\wedge} 2\right)\right) /\left(1+2^{*}[\mathrm{~A}] / 1000\right)-5 / 10^{\wedge} 8\right]-$ matrix of Avogadro.

Field of inverse entropy $[\mathrm{DA}]=10 /[\mathrm{NA}]-$ atomic mass matrix of Dalton. 
The above set of quantum eigenfunctions was obtained by hyperbolic compressing truncated digital sequences $\mathrm{PI}=3.14 \ldots>$ and $\mathrm{E}=2.71 \ldots>$ into infinitesimal space $\left[1 / \mathrm{PI}^{\wedge} 129 \ldots 1 / \mathrm{E}^{\wedge} 148\right]=1 / 10^{\wedge} 64$. This is a natural arithmetical limit of accuracy of root extraction because $1 / \mathrm{PI}^{\wedge} 130=0=1 / \mathrm{E}^{\wedge} 149$.

The quantum eigenvectors $\mathrm{R}(\mathrm{Ai})$ and $\mathrm{A}(\mathrm{Ri})$ were derived analytically using recursive (forward-back, updown, left-right) and mutual transformations of a circle, ellipse, ring, oval, cylinder, cone, sphere, hoop, paraboloid, hyperboloid, ellipsoid, ovaloid in their functional dependence on temporal polynomials and temporal exponents. As a result, we obtained thirteen nodes of standing waves (superposition points) and intermediate spaces for traveling waves ("black holes" of information) for various aggregate states of matter - an ideal crystal [AL...A4], an ideal liquid [AS...A1], an ideal gas [RK...RC], an absolute vacuum $[A X]$. Intermediate spaces $[A 1 \ldots A L],[A(R C) \ldots A S],[A X \ldots A(R K)]$ describe liquid crystal, liquefied gas and partial vacuum. For illustration and comparison with SI-2019 metric values, the partial quantum matrices of Avogadro N(Ai), Planck $\mathrm{P}(\mathrm{Ai})$, Boltzmann $\mathrm{KB}(\mathrm{Ri})$ are shown on the right.

Quaternion of ideal solid state, where B $=602214183$ :

$$
\begin{array}{ll}
\mathrm{A} 4=4 / 137-3^{\star}\left(\mathrm{pi}^{*} \mathrm{e} / 100\right)^{\wedge} 2 . & \mathrm{N}(\mathrm{A} 4)=6.02214100258192270000 \\
\mathrm{AH}=1 / 16 / \mathrm{pi} / \mathrm{e} . & \mathrm{N}(\mathrm{AH})=6.02214100539028840000 \\
\mathrm{NB}=\mathrm{B} /\left(1+4^{*} \mathrm{pi} / 10^{\wedge} 8\right) / 10^{\wedge} 8 . & \mathrm{N}(\mathrm{AB})=6.02214107323543381769 \\
\mathrm{AL}=1 /\left[\operatorname{Ln}(\mathrm{e})+59^{*} \operatorname{Ln}(10)\right] . & \mathrm{N}(\mathrm{AL})=6.02214114501517300000
\end{array}
$$

Quaternion of ideal liquid state:

$$
\begin{aligned}
& A 1=1 / 137 . \\
& A F=1 /\left(137+36 / 10^{\wedge} 3\right) . \\
& A 0=\left(P I^{\star} E / 100\right)^{\wedge} 2 . \\
& A S=1 / 100 /[10 /(10-1)]^{\wedge} 3 .
\end{aligned}
$$

$$
\begin{aligned}
P 1 & =6.62607100557550050000 \\
P F & =6.62607066502366300000 \\
P 0 & =6.62606983982545790000 \\
P S & =6.62606935923704950000
\end{aligned}
$$

Quaternion of ideal gas state, where $B S=\operatorname{Lim}\left\{\operatorname{Sum}\left[B / 10^{\wedge}\left(3^{\star} N+8\right)\right]\right\}=0.00602817$ :

$$
\begin{aligned}
& R C=\left(R+4^{\star} \mathrm{P} I^{\star} C / 10^{\wedge} 10\right) / 10^{\wedge} 8 . \\
& R E=(R+1 / E) / 10^{\wedge} 8 . \\
& R A=[R+1 /(E+A S)] / 10^{\wedge} 8 . \\
& R K=[R+1 /(E+A S+B S)] / 10^{\wedge} 8 .
\end{aligned}
$$$$
\begin{aligned}
& \mathrm{KBC}=1.38064845028400000000 \\
& \mathrm{KBE}=1.38064845018800000000 \\
& \mathrm{KBA}=1.38064845017700000000 \\
& \mathrm{KBK}=1.38064845016800000000
\end{aligned}
$$

Zero-point of ideal vacuum state:

$$
A X=5 / X-1
$$

Two space-time units - the rational one $C=\left(R / 10^{\wedge} 8+4^{*} \mathrm{pi}^{*} \mathrm{C} / 10^{\wedge} 18\right)^{\wedge} 64^{*} 10^{\wedge} 7$ and the transcendental one $\mathrm{K}=(\mathrm{e}+\mathrm{AS}+\mathrm{BS})-$ determine the upper and lower boundaries of the progressive velocity of waves of matter in an ideal gas environment, where any real or any virtual structural element (molecule, atom, nucleon, electron, photon) has 12 absolutely identical neighbors, symmetrically located on the surface of the sphere relative to the its center (like a three-dimensional star with twelve rays). The time shift of ideal waves "sine" and "cosine", recursively moving along the sphere perimeter relative to 12 equidistant points, gives us the angular and phase Boltzmann metrics. By geometrical analogy, such a metric can be transferred to outer space for satellites, planets, stars, constellations, galaxies. 
There are no single quantum units for different aggregate states of matter. Thus, there can be no single theory of the universe, but there are seven interconnected "languages" of science - analytical chemistry (integral calculus of vector-tensors), quantum physics (differential calculus of vector-tensors) and five branches of elementary mathematics - arithmetic, geometry, trigonometry, algebra, logarithms. For the logical connection of these languages, only the Gaussian information functional of the entropy of normal distribution Sqrt $\left(2^{*} \mathrm{pi}^{*} \mathrm{e}\right)$ is sufficient, and mathematical physics in general can be defined simply as the differential geometry of an arbitrarily moving sphere with radius e/2 within the sphere of radius pi/2.

\section{QUANTUM WEB OF GRAVITY}

Using quantum eigenfunctions, eigenvalues, eigenvectors and boundary conditions, we can exactly (up to $\left.1 / 10^{\wedge} 64\right)$ calculate the perimetral matrices of Planck [P], radial matrices of Dirac $[R]$, entropy matrices of Avogadro [NA], atomic mass matrices of Dalton [DA], translation velocity matrices of Maxwell [V], temperature matrices of Kelvin [T], phase matrices of Boltzmann [KB] but let us restrict ourselves only by Newton's gravity $[G]=[P]^{*}(1+[A])-$ matrix, which one for the first time in engineering practice will be presented in its absolute entirety. One of the partial values $\mathrm{G}(\mathrm{NB})$ was not specially calculated to show that the informational entropy in metrology SI principally cannot be less than $1 / 10^{\wedge} 8$, and this is the natural arithmetical limit of accuracy for the unified quantum metric and computing. Previous metrology with declared experimental entropy of fundamental quantum constants was more "honest" from the point of view of mathematical logic and information theory.

Three-dimensional (volume integrals and trigonometry) gravitational matrix:

$$
\begin{aligned}
& G(A 4)=6.6745704910750265485376520841945674109436315576651411126710705788 \\
& G(A H)=6.6745689043376525769289720333765430722301010754854776960339352496 \\
& G(N B), \text { where NB }=B /\left(1+4^{*} \mathrm{pi} / 10^{\wedge} 8\right) / 10^{\wedge} 8 \text { is the upper point of the three-dimensional entropy } \\
& G(A L)=6.6744900157701819328593530509858699615007788098243815678024821509
\end{aligned}
$$

Two-dimensional (surface integrals and planimetry) gravitational matrix:

$$
\begin{aligned}
& G(A 1)=6.6744364873680224292185910483000481884177319277195916202766912038 \\
& G(A F)=6.6744234384921214159611943953611634645758144315309572175422129523 \\
& G(A 0)=6.6743918194962957193271732313472748453752700748755734914847060150 \\
& G(A S)=6.6743734048658876250224876184710519248528280242449461899834781806
\end{aligned}
$$

One-dimensional (contour integrals and algebra) gravitational matrix:

$$
\begin{aligned}
& G(R C)=6.6739140452062992860293936167978444193636663660125695249577923340 \\
& G(R E)=6.6739140244578731661939937274667944631317509843397120609727937384 \\
& G(R A)=6.6739140215288578693056001955271762539009208673810926887358535804 \\
& G(R K)=6.6739140195795574401178024788041027602493412757457590964306919405
\end{aligned}
$$

Zero-dimensional point (circle center) of the gravity web:

$$
G(A X)=6.6725781076198223768643093336888740997069675170845242464300377157
$$

The complete set of radial gravity matrices presented here was obtained analytically for the first time in engineering practice. 


\section{DISCUSSION}

The web of gravity fully harmonizes classical mechanics with quantum mechanics, and Newton's theory of gravity with Einstein's general theory of relativity within the framework of objective logic of common sense, according to which the experimentally detected one-dimensional (radial) temperature field and two-dimensional (transverse ring) electromagnetic field - these are only part of special cases of the mathematical description of wave processes in a three-dimensional gravitational field. Gravitational waves (density waves of the translational velocity field) are primary in relation to electrical and thermal ones and cannot be described by only electrodynamics and thermodynamics, the combination with chromodynamics is required - and this is already the relative dynamics of frequency and wavelength, showing the relationship between transcendental wave functions "pi" and "e".

The eigenvectors for the ideal gas state of matter make it possible to accurately calculate the weighted average temperature of the cosmic microwave background radiation TBG or the so-called "relic" cosmic temperature, which is measured by the COBE satellite in the interval $(2.72491 \ldots 2.72605) \mathrm{K}$.

$$
T B G=\operatorname{Median}\{\text { Median }[\mathrm{e} \ldots(\mathrm{e}+\mathrm{AS})] \ldots \text { Median}[(\mathrm{e}+\mathrm{AS}) \ldots \mathrm{K}]\},
$$

where the operator "Median" means the arithmetic mean of the four mean values - root mean square, arithmetical mean, geometrical mean and harmonic mean.

$\mathrm{TBG}=2.7252543275634558348257203374492224585435413056374625751755012329$.

This is the first in engineering practice direct calculation the cosmic background temperature without any preliminary measurements and this is a direct confirmation of the mathematical definition of gravity as the relative amplitude-phase and frequency-wavelength density of the translational velocity field.

\section{CONCLUSION}

A superposition of dynamic and infinitely long ideal wave functions "sine" and "cosine" doubles the observed frequency of oscillations when the instant translational velocity " $v$ " tends to $C$ - this was first shown and stated by Nyquist, but intuitively clear for any electrical engineer - as a natural result of fullwave rectification of alternative current. Thus, the canonic expression for the kinetic energy of quanta of different frequency ranges should be as follows in physical terms of chromodynamics (instant action and frequency) and of gravidynamics (instant mass and velocity):

$$
\mathrm{QE}=\mathrm{h}^{*} \mathrm{~F}^{*}\left[1+(\mathrm{v} / \mathrm{C})^{\wedge} 2\right]=\mathrm{m}^{*} \mathrm{v}^{\wedge} 2 / 2^{*}\left[1+(\mathrm{v} / \mathrm{C})^{\wedge} 2\right]
$$

Moreover, now we can write down the canonic expressions for the kinetic energy of quanta in terms of electrodynamics (instant charge and voltage) and thermodynamics (instant phase and temperature) as the natural result of one half-wave rectification of alternative current:

$$
\mathrm{QE}=\mathrm{q}^{\star} \mathrm{U} /\left[1+(\mathrm{v} / \mathrm{C})^{\wedge} 2\right]=\mathrm{k}^{\star} \mathrm{T} /\left[1+(\mathrm{v} / \mathrm{C})^{\wedge} 2\right]
$$

The energy of quanta tends to the Einsteinian value $\mathrm{m}^{*} \mathrm{C}^{\wedge} 2$ if and only if the translational velocity tends to $\mathrm{C}$, and tends to Newtonian zero if and only if the velocity tends to zero. But in a physically observable real and in mathematically described virtual Universe there can never be zero velocity, zero acceleration, zero 
momentum, zero force, zero mass, zero charge, zero energy, zero entropy and zero information. Just as in recursive arithmetic there is no absolute zero, so in natural science can be no universal field theory. World Wide Web of Gravity is there - the mathematical quintessence of the classical Newtonian physics.

\section{REFERENCES}

1. Мачуський Є. «Електрон»/ ISBN 978-966-622-572-9/ Київ, НТУУ «КПІ», 2013.

2. Machusky E.A. "Logarithmetic of Quantum Physics"/ $16^{\text {th }}$ Int. Sc. M.Kravchuk Conf., vol.2 "Algebra. Geometry. Analysis"/ Kyiv, NTUU “KPI”, May 14-15, (2015).

3. Мачуський Є.А «Дифреренціальна альфа-система числення в обернених кільцях»/ V Міжнар. наук.-практ. конф. «Методи та засоби кодування, захисту й ущільнення інформації»/ Вінниця, 19-21 квітня, (2016).

4. Eugene Machusky "Demystification of Quantum Physics"/ script and film by Lyubov Lisovska www.youtube.com/ August 26, (2016).

5. Eugene Machusky “Analytics of Quantum Physics or Quantum Language of Universe"/ Workshop Quantum-2017/ Torino, Italy, May 7-13, (2017).

6. Eugene Machusky "Quantum Metric of Classic Physics"/ IOP conf. series: Material Science and Engineering/ Online ISSN: 1757-899X, vol. 239, (2017).

7. Eugene Machusky "Quantum Physics as Dynamic Space-Time Differential Calculus"/ $3^{\text {rd }}$ Int. Conf. on Theoretical and Condensed Matter Physics/ New York, USA, October 19-21, (2017).

8. Eugene Machusky "Complex Geometry of Wave Motion"/ Int. J. of Engineering and Technology, v.10, no.2, (2018).

9. Eugene Machusky "Natural Qubit Matrix of Primary Elements of Matter"/ MATEC web of Conferences, 186, 01005, (2018).

10. Eugene Machusky "Quantum Alphabet of Matter Language"/ Int. Conf. on Material Science and Engineering/ Rome, Italy, June 25-26, (2018).

11. Eugene Machusky "Transfinite Wave Mathematics of Relative Space-Time"/ VII Міжн. наук.-пр. конф. «Обробка сигналів і негаусівських процесів»/ Черкаси, Україна, 23-24 травня (2019).

12. Eugene Machusky "Quantum Information Paradigm of Physical Reality"/ Global Cyber Security Forum 2019/ Харків, Україна, 14-16 листопада, (2019).

13. Eugene Machusky "A Quantum Melody and Painting of Dancing Electron"/ E.Machusky, O.Goncharov, Sofiia Mikadse, Salome Mikadse/ Research J. of Nanoscience and Engineering, ISSN 2637-5591, v.4, iss.1, (2020). 
